\title{
Photoelectron spectroscopy of laser-dressed atomic helium
}

\author{
Severin Meister $\odot,{ }^{1, *}$ Aaron Bondy, ${ }^{2,3}$ Kirsten Schnorr, ${ }^{4}$ Sven Augustin, ${ }^{4}$ Hannes Lindenblatt $\odot,{ }^{1}$ Florian Trost $\odot,{ }^{1}$ \\ Xinhua Xie, ${ }^{4}$ Markus Braune $\odot,{ }^{5}$ Rolf Treusch $\odot,{ }^{5}$ Bastian Manschwetus, ${ }^{5}$ Nora Schirmel,${ }^{5}$ Harald Redlin, ${ }^{5}$ Nicolas Douguet, ${ }^{6}$ \\ Thomas Pfeifer, ${ }^{1}$ Klaus Bartschat $\odot,^{2}$ and Robert Moshammer ${ }^{1}$ \\ ${ }^{1}$ Max-Planck-Institute for Nuclear Physics, 69117 Heidelberg, Germany \\ ${ }^{2}$ Department of Physics and Astronomy, Drake University, Des Moines, Iowa 50311, USA \\ ${ }^{3}$ Department of Physics, University of Windsor, Windsor, Ontario N9B 3P4, Canada \\ ${ }^{4}$ Paul Scherrer Institute, 5232 Villigen, Switzerland \\ ${ }^{5}$ Deutsches Elektronen-Synchrotron, 22607 Hamburg, Germany \\ ${ }^{6}$ Department of Physics, Kennesaw State University, Marietta, Georgia 30060, USA
}

(Received 18 September 2020; accepted 23 November 2020; published 8 December 2020; corrected 22 December 2020)

\begin{abstract}
Photoelectron emission from excited states of laser-dressed atomic helium is analyzed with respect to laser intensity-dependent excitation energy shifts and angular distributions. In the two-color extreme ultraviolet (XUV)-infrared (IR) measurement, the XUV photon energy is scanned between $20.4 \mathrm{eV}$ and the ionization threshold at $24.6 \mathrm{eV}$, revealing electric dipole-forbidden transitions for a temporally overlapping IR pulse $\left(\approx 10^{12} \mathrm{~W} \mathrm{~cm}^{-2}\right)$. The interpretation of the experimental results is supported by numerically solving the timedependent Schrödinger equation in a single-active-electron approximation.
\end{abstract}

DOI: 10.1103/PhysRevA.102.062809

\section{INTRODUCTION}

Photoelectron spectroscopy is a powerful technique to obtain compositional and structural information about matter and to investigate light-matter interactions in general. It has been successfully employed and continuously developed over many decades in atomic and molecular physics [1]. Photoelectrons carry information about the electronic bound and continuum states of the corresponding atom, as well as information about the absorbed and emitted photons.

With the advent of intense optical lasers, multiphoton absorption in atoms and molecules became feasible, enabling the observation of a variety of new phenomena, e.g., multiphoton excitation microscopy [2], resonance-enhanced multiphoton ionization (REMPI) [3], Doppler-free two-photon spectroscopy [4,5], and high-order harmonic generation (HHG) $[6,7]$, to name just a few. One step further in the investigation of light-matter interactions is the implementation of two-color ionization and excitation schemes, which reveal laser-induced continuum structures [8] and light-induced structures (LIS) [9]. In the former case, the dressing laser field couples bound states to the continuum, giving rise to a resonant structure [10-13]. In the latter case, the ground state is coupled to

*severin.meister@mpi-hd.mpg.de

Published by the American Physical Society under the terms of the Creative Commons Attribution 4.0 International license. Further distribution of this work must maintain attribution to the author(s) and the published article's title, journal citation, and DOI. Open access publication funded by the Max Planck Society. excited states beyond the one-photon allowed dipole transition via two-color photoexcitation [14-17].

In this paper, we report the use of XUV (extreme ultraviolet) radiation with tunable wavelength provided by the free-electron laser in Hamburg (FLASH) in combination with a synchronized infrared (IR) laser to obtain a detailed picture of excited states in laser-dressed atomic helium. The extreme ultraviolet (XUV) photon energy is scanned over the 1 snp ${ }^{1} P$ Rydberg excitation series to a value just below the ionization threshold. The superimposed IR pulses ( $800 \mathrm{~nm}$ wavelength) arrive with a freely adjustable time delay with respect to the excitation pulses. Their intensity is too low to ionize $\mathrm{He}$ in its ground state, but strong enough to ionize it from excited states that are temporarily reached via a combination of XUV and IR photons. The ionization yield and angular distributions are analyzed as a function of the XUV photon energy, the IR time delay, and the IR intensity. In the case of temporally overlapping pulses, by absorption of one XUV and one or more IR photons, one electron is lifted from the ground into a continuum state through laser-dressed excited states, including those that are not accessible by pure single-photon excitation. The interpretation of the experimental results is supported by numerical calculations based on the time-dependent Schrödinger equation (TDSE) within the single-active electron (SAE) approximation.

This two-color scheme has the clear advantage over, e.g., single-color REMPI setups, where the dominant contribution to the excitation energy is delivered by only one XUV photon, and hence the laser intensity can be kept low. Therefore, field-induced changes to energy levels and fragmentation are minimized. This opens up precision-spectroscopic studies of atoms and molecules under less-perturbing conditions. 
There are photoionization measurements employing HHGbased XUV radiation in combination with IR laser radiation [18-20]. However, these experiments rely on single-photon excitation of 1 snp ${ }^{1} P$ states and the following ionization through IR photon absorption. In contrast, the presented measurement focuses on multiphoton excitation, enabled by the combined interaction of XUV and IR photons.

\section{EXPERIMENTAL AND THEORETICAL METHODS}

The experiment was carried out with the reaction microscope (REMI) end station [21,22] at the free-electron laser (FEL) FLASH2 [23,24]. FLASH2 features variable-gap undulators that allow us to quickly change the photon wavelength [25] over a broad spectral range. During the measurements, the XUV photon energy was scanned in steps of $0.2 \mathrm{eV}$ from $20.4 \mathrm{eV}$ to just below the ionization threshold of atomic helium at $24.6 \mathrm{eV}$ [26]. The FEL pulse-length was about $40 \mathrm{fs}$ full-width at half maximum (FWHM) in intensity, and the pulse energy $(<10 \mathrm{~nJ})$ was reduced to a level such that two-XUV-photon absorption in He can be neglected. Synchronized, but with a timing jitter of several ten femtoseconds, the IR probe laser $(800 \mathrm{~nm})$ was superimposed collinearly with the XUV beam. In order to ensure optimal temporal overlap with the FEL pulses, an IR pulse-duration of about $90 \mathrm{fs}$ FWHM was chosen. The IR pulse energy and the focusing conditions were adjusted such that intensities in the order of up to $10^{13} \mathrm{~W} \mathrm{~cm}^{-2}$ were reached in the target. With a diameter of about $30 \mu \mathrm{m}$, the focus of the IR beam was significantly wider than the FEL focus $(\approx 10 \mu \mathrm{m})$. XUV and IR radiation were linearly polarized and aligned parallel to each other. Both beams were focused onto a dilute supersonic gas jet of atomic helium in the center of the REMI, which is equipped with two time- and position-sensitive detectors [27] to collect all charged fragments (electrons and ions) within the full $4 \pi$ solid angle. Time-of-flight and position information is used to retrieve the particles' momentum vectors at the time of ionization.

During the XUV photon-energy scans, the FEL pulse energy and the FEL beam diameter change slightly. Together with the energy-dependent absorber-foil transmissions and mirror reflectivities, this leads to small variations in the photon flux. In our analysis, these effects are corrected by normalizing the data for each XUV energy with the simultaneously recorded yield of $\mathrm{H}_{2}^{+}$ions, which stem from a constant and weak background of $\mathrm{H}_{2}$ gas in the REMI chamber. The ionization cross section of $\mathrm{H}_{2}$ was taken from Ref. [28].

In the experiment, two sets of data were taken, one with an IR intensity of approximately $I_{\text {high }} \approx 8 \times 10^{12} \mathrm{~W} \mathrm{~cm}^{-2}$ and one with $I_{\text {low }} \approx 1 \times 10^{12} \mathrm{~W} \mathrm{~cm}^{-2}$. We note that the temporal jitter between FEL and IR, which is comparable to the IR pulse duration, leads effectively to a lowering of the average IR intensity for the case of overlapping pulses. The influence of this imperfect overlap of both pulses increases with the IR intensity in the same way as the contribution of nonlinear multiphoton transitions increases. Therefore, in the comparison to theory, a smaller difference in intensity between the low and high IR intensity case was chosen in our calculations in order to mimic the corresponding experimental conditions.
The theoretical part of this study is based on numerically solving the TDSE in the SAE approximation. Electrons are assumed to be noninteracting, while the ground state is effectively described as a $1 s 1 s^{\prime}{ }^{1} S$ state, where the $1 s$ is close to the $\mathrm{He}^{+}$orbital and the $1 s^{\prime}$ is treated like a valence orbital. As always in theory, the binding energies of the $n \ell$ valence electrons are not exact. While 1 sn $\ell$ Rydberg states with angular momenta $\ell \geqslant 2$ have very accurate binding energies, this is not quite the case for $p$ electrons and particularly for $s$ electrons, due to the small or missing centrifugal barrier. Since excitation energies are measured from the ground state, much of the remaining discrepancies are due to the binding energy of the $1 s^{\prime}$ orbital.

Specifically, we used the same one-electron potential as Birk et al. [29],

$$
V(r)=-\frac{1}{r}-\left(\frac{1}{r}+1.3313\right) \exp (-3.0634 r),
$$

where $r$ is the distance from the nucleus, to calculate the valence orbitals. The difference of excitation energies compared to the recommended excitation from the NIST database [26] is less than $0.2 \mathrm{eV}$ even in the worst-case scenario and does not alter the essential conclusions presented below. We will sometimes omit the inner $1 s$ electron to simplify the notation, keeping in mind that only two-electron singlet spin states are accessible, since spin-forbidden transitions are negligible.

The laser parameters were chosen according to the available knowledge regarding the actual experimental conditions. The XUV pulse duration was taken as $40 \mathrm{fs}$ (FWHM value of a peak intensity of $1 \times 10^{12} \mathrm{~W} / \mathrm{cm}^{2}$ with a Gaussian envelope) and the IR pulse duration as $80 \mathrm{fs}$. While the XUV photon energy was varied over a range in steps of $0.05 \mathrm{eV}$, the central IR photon energy was held fixed at $1.55 \mathrm{eV}(800 \mathrm{~nm})$. Since both beams are linearly polarized along the same direction, the initial state can be propagated very efficiently and accurately. Specifically, we used an updated version of the code described by Douguet et al. [30].

\section{RESULTS}

Over the XUV scanning range, the helium atom can be excited from the $1 s^{2}{ }^{1} S$ ground state to a $1 s n p{ }^{1} P$ excited state for specific XUV photon energies, according to the electricdipole selection rules. The excited atom can be ionized by absorbing one or more IR photons ( $\hbar \omega=1.55 \mathrm{eV}$ ) of a subsequent laser pulse, promoting the weakly bound electron into the continuum. This mechanism is depicted in an energy-level scheme in the upper part of Fig. 1.

Experimental data for the corresponding process are shown in Fig. 2, where the yield of photoelectrons is plotted against the XUV photon energy for a nonoverlapping temporally delayed IR pulse with intensity $I_{\text {high }}$. Clearly visible are the yield enhancements for XUV energies that match the 1 snp ${ }^{1} P$ excitation energies in helium ( $2 p$ at $21.2 \mathrm{eV}, 3 p$ at $23.1 \mathrm{eV}, 4 p$ at $23.7 \mathrm{eV}$, etc. [26]). Also shown in Fig. 2 is the photoelectron yield for XUV and IR pulses in temporal overlap (yellow distribution). Compared to delayed ionization, the ${ }^{1} P$-excitation peaks remain while an additional maximum appears around $22.4 \mathrm{eV}$. This feature was observed and described in transientabsorption measurements as a LIS $[9,15,16,31]$. As XUV 


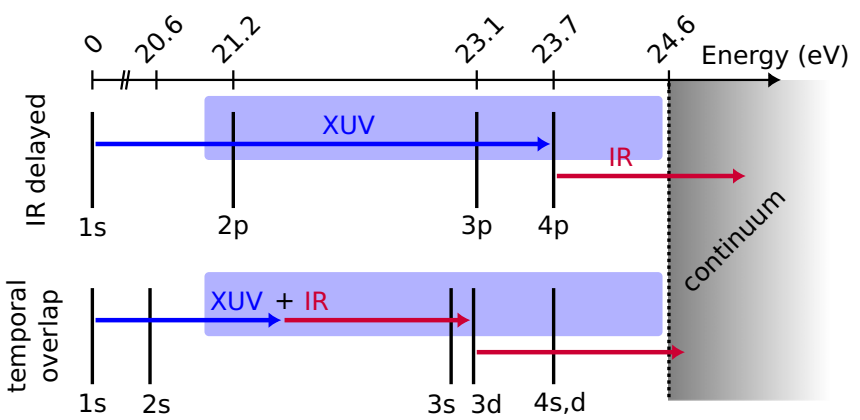

FIG. 1. Electronic energy level scheme of helium with different ionization pathways through intermediate excited states. The XUV energy scanning range is indicated by the blue box.

and IR radiation are simultaneously present, the helium atom can undergo dipole-forbidden (for single photons) transitions, provided the XUV photon absorption is accompanied by the absorption or emission of IR photons of the dressing laser field. In the simplest and dominant case, one XUV photon and one IR photon combined drive $1 s^{2} \rightarrow 1 s n s$ or $1 s^{2} \rightarrow 1 s n d$ transitions. By absorbing additional IR photons, the excited atom is ionized.

This mechanism is depicted in the lower part of Fig. 1. Direct two-photon absorption couples the ground state to 1 sns and 1 snd states. In contrast to $P$-state ionization, peaks of LIS emerge at XUV energies matching the energy of the excited state plus or minus one IR photon.

In Fig. 3, the measured yield distributions for overlapping pulses are shown for the two cases of low and high IR intensity. The purple distribution in the background is recorded with $I_{\text {low }}$, while the yellow distribution is again for the significantly larger intensity $I_{\text {high. }}$. The latter exhibits a gradual decrease in the yield from the peak corresponding to the $2 p$ state at $21.2 \mathrm{eV}$ over the $3 p$ state at $23.2 \mathrm{eV}$ up to

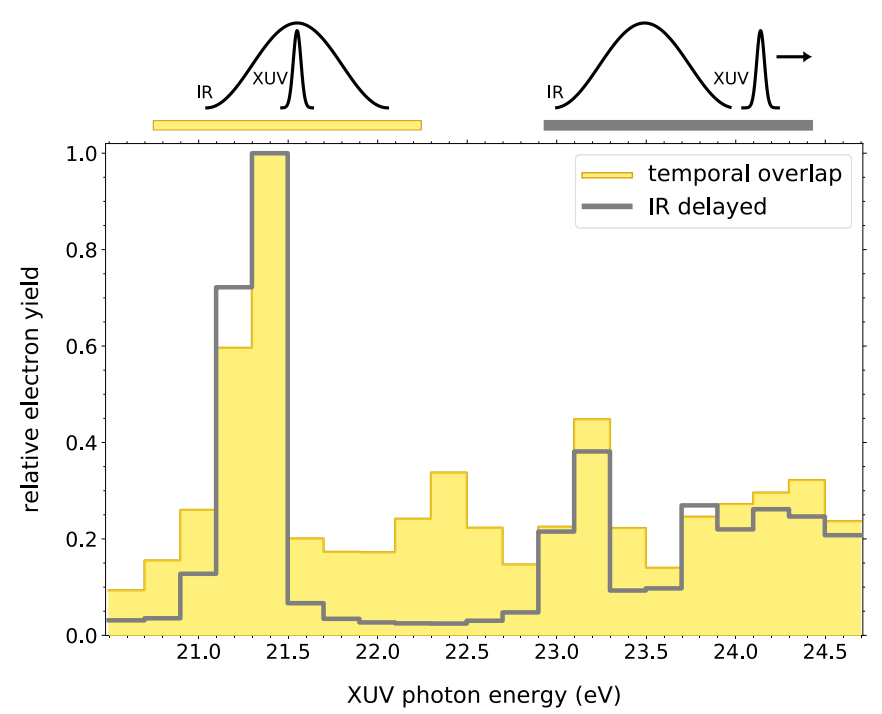

FIG. 2. Photoelectron yield measured for two different delays between the XUV and IR pulses. Both curves are normalized to their maximum yield, and statistical error bars are much smaller than the line thickness. the higher $n p$ states (not visible due to the resolution). This overall decrease with rising XUV energy can be explained by the energy dependence of the cross section for the excitation step [32]. The excitation probabilities are directly mapped to the ionization yield in the case of $I_{\text {high }}$, where the IR intensity is large enough to ionize all excited states independent of the number of photons $(N)$ needed. For low IR intensity (purple distribution in Fig. 3), on the other hand, the scaling law $R \sim I^{N}$ of the transition rate $R$ with the laser intensity $I$ in multiphoton processes becomes relevant [33]. Therefore, the ionization yield of the $2 p$ state, which requires $N=3$ IR photons, is reduced compared to the yield from the $3 p$ and higher $n p$ states $(N=1)$.

Two approaches are employed to assign the LIS to specific field-free atomic excited states. First, we analyze the calculated distributions for excitation in combination with ionization and compare them with the experimental ionization yield. Second, the inspection of the measured photoelectron angular distribution allows us to deduce the intermediate bound state that the electron was emitted from.

Figure 4 shows theoretical predictions for XUV and IR pulses in temporal overlap. The calculated ionization probability is plotted against the XUV photon energy for two different IR intensities, color coded in yellow and purple for high and low IR intensity, respectively (similar to the measurement shown in Fig. 3). In addition to the ionization yield, our calculations predict the population distribution of excited atomic states at the end of the pulses as a function of the XUV energy and the IR intensity. The most prominently populated states in the theoretical excitation-probability distributions are marked by arrows in Fig. 4. They serve as an indicator of the role of the respective excited states and their population (either direct or light-induced) at a given XUV energy en route to ionization. This allows assignment of the peaks in the ionization probability distribution (bottom panel of Fig. 4) to the excited states from which the atom is ionized.

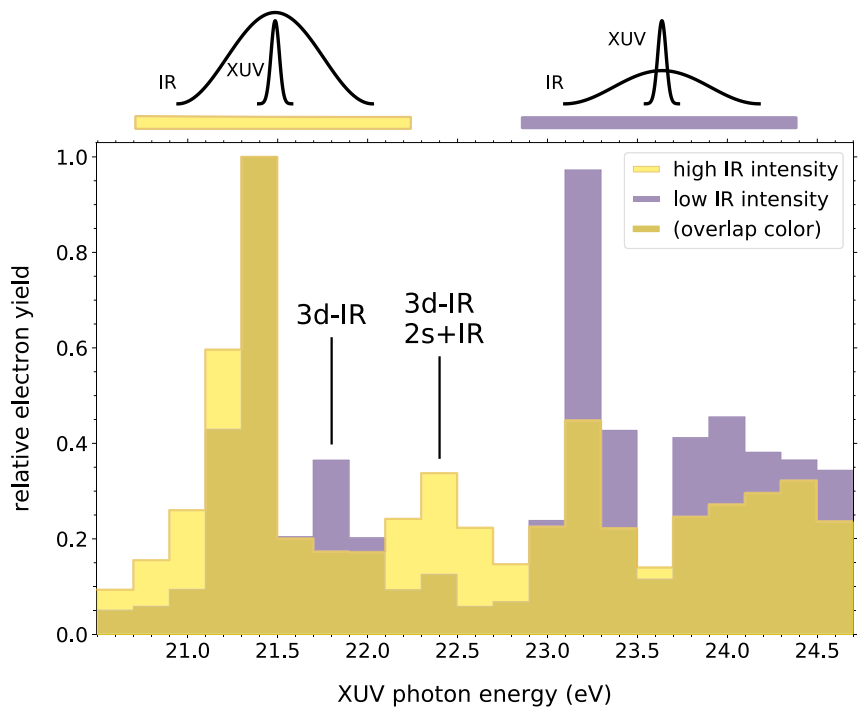

FIG. 3. Photoelectron yield measured for temporally overlapping XUV and IR pulses. The distribution for high IR intensities ( $\left.I_{\text {high }}\right)$ is shown in yellow (light gray), and the distribution for low IR intensities $\left(I_{\text {low }}\right)$ in purple (dark). 


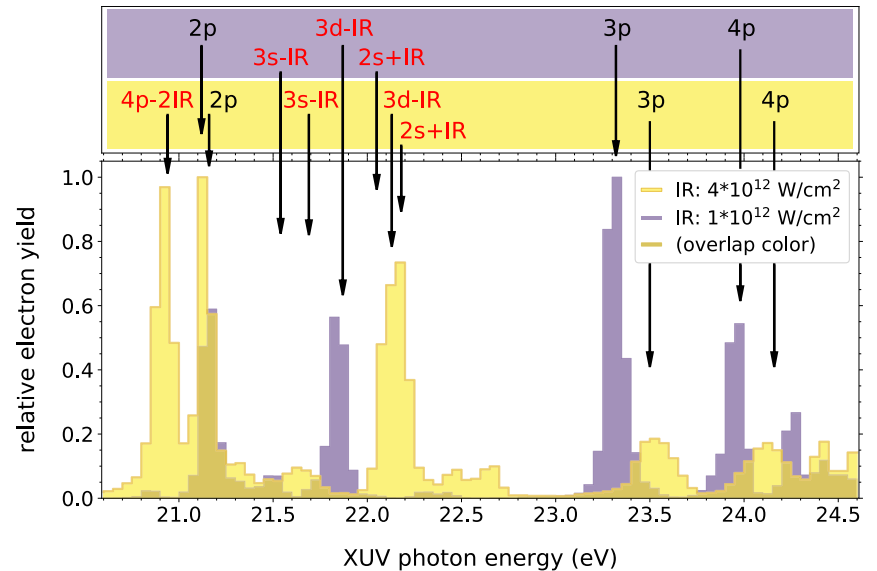

FIG. 4. Calculated ionization probability for two different IR intensities. Arrows in the top panel indicate the calculated positions of excited (black) and light-induced (red) states. + (-) IR denotes the emission (absorption) of an IR photon.

Electronic energy levels experience an AC Stark shift due to the IR laser field [34,35]. This shift is clearly seen for the $3 p$ state in theory (see Fig. 4), but is much smaller in the experiment (see Fig. 3). However, experimental Stark shifts can be seen in the photoelectron kinetic energy spectrum (not shown here). Overall, we see good agreement between experiment and theory when comparing the purple distributions in Figs. 3 and 4. Similar to the experiment, the calculated ionization yield shows a reduced contribution of the $2 p$ state relative to the $3 p$ state when the IR intensity is lowered. Moreover, the strengths and the positions of the LIS peaks, which only appear in temporal overlap, are well reproduced by the calculations. Small shifts in energy are attributed to the already mentioned inherent inaccuracies of electronic binding energies in the SAE model. Most importantly, for low IR intensity the dominating LIS peak is found around $21.8 \mathrm{eV}$ as in the experimental counterpart. As the enhancements by other excited states appear at distinctively different positions and are much less pronounced, this dominating LIS peak at $21.8 \mathrm{eV}$ (purple distribution in Fig. 3) can be assigned to the $3 d$ state.

The situation changes for high IR intensity where the dominant LIS peak is shifted to a larger XUV energy of about $22.4 \mathrm{eV}$ in experiment (yellow in Fig. 3). Comparison with theory indicates that in this case ionization proceeds through the Stark-shifted $3 d$ and $2 s$ excited states. Both contribute to the dominating LIS peak at about $22.2 \mathrm{eV}$ according to our state assignment in Fig. 4. Relative to the $3 d$ state, the $2 s$ contribution becomes more relevant at high intensity because, in order to populate the $2 s$ state, the atom absorbs one XUV photon while emitting one IR photon. Ionization takes place by absorbing another three IR photons. In contrast to this effective four IR-photon transition, ionization via the $3 d$ state involves only two IR photons. We note that the large shift of the $3 d$ LIS with IR intensity seen in the experiment is also consistent with the calculation by Chen et al. [9].

The electronic structure of the dominant LIS involving both the $2 s$ and $3 d$ excited states at $22.4 \mathrm{eV}$ for high IR intensity in Fig. 3 can also be deduced from the photoelectron

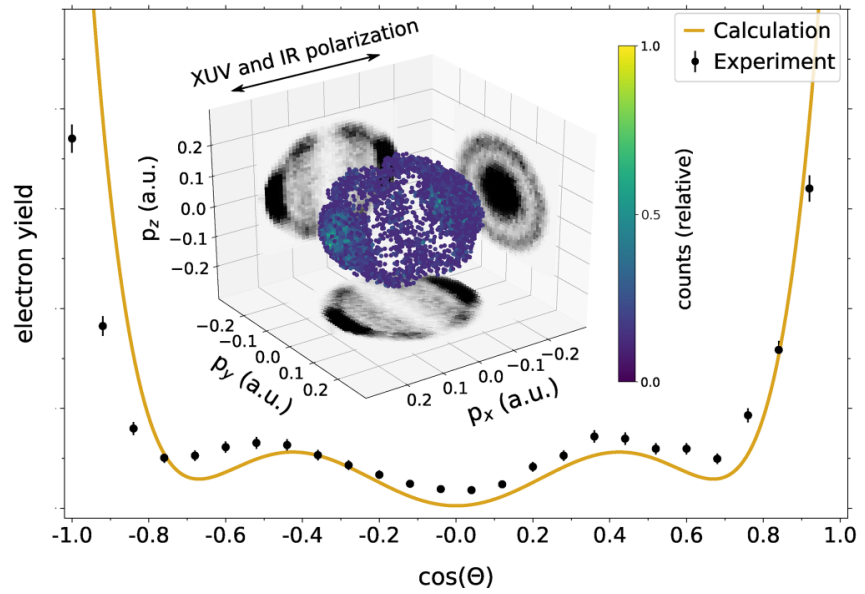

FIG. 5. PAD for the dominant LIS at high IR intensity. (Exp: $E_{\mathrm{XUV}}$ from 22.2 to $22.5 \mathrm{eV}$ and $E_{\mathrm{e}}$ from 0.2 to $0.5 \mathrm{eV}$, Calc: $E_{\mathrm{XUV}}$ from 22 to $22.3 \mathrm{eV}$ and $E_{\mathrm{e}}$ from 0.2 to $0.5 \mathrm{eV}$ ). The experimental counts are normalized to theory. The inset shows the corresponding measured 3D photoelectron momentum distribution.

angular distribution (PAD). This is shown in Fig. 5 where the yield of electrons is plotted as a function of their emission angle $\Theta$ with respect to the laser polarization axis.

The distribution exhibits a typical " $F$-like" shape, indicating the angular quantum number of the continuum state to be $L=3$. Starting from a ${ }^{1} S$ state $(L=0)$, an angular quantum number of $L=3$ can only be reached by absorbing at least three photons. For an atom in a ${ }^{1} \mathrm{D}$ state $(L=$ 2 ), absorbing one IR photon is sufficient to obtain an $F$ like PAD. One can deduce the populated bound states by taking the photoelectron kinetic energy $(0.4 \mathrm{eV})$ minus the IR photon energies (each $1.55 \mathrm{eV}$ ) while also accounting for the Stark-shifted continuum level. One finds that the matching states are the $3 d$ and $2 s$ excited states. The yellow solid line in Fig. 5 shows the calculated PAD for the considered LIS. We find very good agreement between experiment and theory, thereby supporting our interpretation of the mechanism.

The three-dimensional PAD of the dominant LIS is also contained in the measured three-dimensional photoelectron momentum distribution, shown in the inset of Fig. 5. Dots in the plot represent a bin in momentum space, with the yield within each bin being color coded. The energy range was chosen from 0.3 to $0.5 \mathrm{eV}$, resulting in a spherical shell of dots in the plot. The projections on the walls show the integrated yield along one specific direction. The two small maxima in Fig. 5 are found as two rings around the polarization axis, while the large maxima are found in the three-dimensional distribution for maximal absolute $p_{x}$ momentum, i.e., along the polarization axis of IR and XUV.

\section{CONCLUSION}

We have measured and analyzed photoelectrons stemming from laser-dressed atomic helium. The helium atoms were photo-excited over a large energy range by XUV FEL radiation in the presence of a moderately strong IR laser $(\approx$ $10^{12} \mathrm{~W} \mathrm{~cm}^{-2}$ ). The scheme allowed us to reveal light-induced 
structures alongside the ${ }^{1} P$ Rydberg series. The observed excitation energies of the LIS for varying IR intensities were supported by TDSE calculations. The photoelectron angular distribution and the kinetic energy were used to assign the LIS. The dominant LIS in the photoelectron yield of laserdressed helium was identified as stemming from the $1 s 3 d^{1} D$ excited state for an IR intensity of about $1 \times 10^{12} \mathrm{~W} \mathrm{~cm}^{-2}$, while for a higher IR intensity of about $4 \times 10^{12} \mathrm{~W} \mathrm{~cm}^{-2}$ the $1 s 2 s{ }^{1} S$ state also contributes significantly.

Our investigation complements previous transientabsorption measurements on light-induced structures but brings up additional aspects. While transient-absorption measurements reveal LIS in the absorption spectrum without the need for ionization, our measurement is sensitive to the excited states from which electrons are emitted and allows us to measure and assign angular distributions of the corresponding continuum final states.

The presented analysis suggests the preference to populate the $1 s 3 d{ }^{1} D$ state rather than the $1 s 3 s{ }^{1} S$ state. This is can be seen in the high IR intensity case, where all excited states get ionized, so the yield is independent of the number of ionizing photons. We find the propensity of helium in its ground state to increase angular momentum by two-photon absorption, thus driving a bound-bound transition. This can be understood in the context of Fano's propensity rule [36], originally stated between bound and continuum states, and the propensity analysis of continuum-continuum transitions by Busto et al. [37], both stating the propensity to increase the angular momentum in photoabsorption.

\section{ACKNOWLEDGMENTS}

The work was supported, in part, by the U.S. National Science Foundation under Grants No. PHY-1803844 (K.B.) and No. PHY-2012078 (N.D.) and by the XSEDE supercomputer allocation No. PHY-090031 (A.B., K.B., N.D.). The calculations were carried out on Comet at the San Diego Supercomputer Center and Frontera at the Texas Advanced Computing Center. A.B. is grateful for a Michael Smith Scholarship. S.A. has received funding from the European Union's Horizon 2020 research and innovation programme under Marie Skodowska-Curie Grant Agreement No. 701647. We acknowledge DESY (Hamburg, Germany), a member of the Helmholtz Association HGF, for the provision of experimental facilities. Parts of this research were carried out at FLASH.
[1] E. O. Lawrence and M. A. Chaffee, On the direction of emission of photoelectrons from potassium vapor by ultraviolet light, Phys. Rev. 36, 1099 (1930).

[2] A. Diaspro, P. Bianchini, G. Vicidomini, M. Faretta, P. Ramoino, and C. Usai, Multi-photon excitation microscopy, BioMed. Eng. OnLine 5, 36 (2006).

[3] T. Streibel and R. Zimmermann, Resonance-enhanced multiphoton ionization mass spectrometry (REMPI-MS): Applications for process analysis, Annu. Rev. Anal. Chem. 7, 361 (2014).

[4] L. S. Vasilenko, V. P. Chebotaev, and A. V. Shishaev, Line shape of two-photon absorption in a standing-wave field in a gas, ZhETF Pis. Red. 12, 161 (1970) [JETP Lett. 12, 113 (1970)].

[5] C. Wieman and T. W. Hänsch, Doppler-Free Laser Polarization Spectroscopy, Phys. Rev. Lett. 36, 1170 (1976).

[6] P. B. Corkum, Plasma Perspective on Strong Field Multiphoton Ionization, Phys. Rev. Lett. 71, 1994 (1993).

[7] M. Lewenstein, P. Balcou, M. Y. Ivanov, A. L'Huillier, and P. B. Corkum, Theory of high-harmonic generation by lowfrequency laser fields, Phys. Rev. A 49, 2117 (1994).

[8] Y. Heller, V. Lukinykh, A. Popov, and V. Slabko, Experimental evidence for a laser-induced autoionizing-like resonance in the continuum, Phys. Lett. A 82, 4 (1981).

[9] S. Chen, M. J. Bell, A. R. Beck, H. Mashiko, M. Wu, A. N. Pfeiffer, M. B. Gaarde, D. M. Neumark, S. R. Leone, and K. J. Schafer, Light-induced states in attosecond transient absorption spectra of laser-dressed helium, Phys. Rev. A 86, 063408 (2012).

[10] P. Knight, M. Lauder, and B. Dalton, Laser-induced continuum structure, Phys. Rep. 190, 1 (1990).

[11] P. Coleman, P. Knight, and K. Burnett, Laser-induced continuum structure in multiphoton ionisation, Opt. Commun. 42, 171 (1982).
[12] Y. Heller and A. Popov, Parametric generation and absorption of tunable vacuum-ultraviolet radiation controlled by laserinduced autoionizing-like resonances in the continuum, Opt. Commun. 18, 449 (1976).

[13] L. Armstrong, B. L. Beers, and S. Feneuille, Resonant multiphoton ionization via the fano autoionization formalism, Phys. Rev. A 12, 1903 (1975).

[14] M. Chini, X. Wang, Y. Cheng, Y. Wu, D. Zhao, D. A. Telnov, S.-I. Chu, and Z. Chang, Sub-cycle oscillations in virtual states brought to light, Sci. Rep. 3, 1105 (2013).

[15] M. Reduzzi, J. Hummert, A. Dubrouil, F. Calegari, M. Nisoli, F. Frassetto, L. Poletto, S. Chen, M. Wu, M. B. Gaarde, K. Schafer, and G. Sansone, Polarization control of absorption of virtual dressed states in helium, Phys. Rev. A 92, 033408 (2015).

[16] J. E. Bækhøj and L. B. Madsen, Light-induced structures in attosecond transient-absorption spectroscopy of molecules, Phys. Rev. A 92, 023407 (2015).

[17] M. J. Bell, A. R. Beck, H. Mashiko, D. M. Neumark, and S. R. Leone, Intensity dependence of light-induced states in transient absorption of laser-dressed helium measured with isolated attosecond pulses, J. Mod. Opt. 60, 1506 (2013).

[18] P. Ranitovic, X. M. Tong, B. Gramkow, S. De, B. DePaola, K. P. Singh, W. Cao, M. Magrakvelidze, D. Ray, I. Bocharova, H. Mashiko, A. Sandhu, E. Gagnon, M. M. Murnane, H. Kapteyn, I. Litvinyuk, and C. L. Cocke, IR-assisted ionization of helium by attosecond extreme ultraviolet radiation, New J. Phys. 12, 013008 (2010).

[19] N. Mayer, P. Peng, D. M. Villeneuve, S. Patchkovskii, M. Ivanov, O. Kornilov, M. J. J. Vrakking, and H. Niikura, Population transfer to high angular momentum states in infrared-assisted XUV photoionization of helium, J. Phys. B: At., Mol. Opt. Phys. 53, 164003 (2020). 
[20] L. H. Haber, B. Doughty, and S. R. Leone, Continuum phase shifts and partial cross sections for photoionization from excited states of atomic helium measured by high-order harmonic optical pump-probe velocity map imaging, Phys. Rev. A 79, 031401(R) (2009).

[21] S. Meister, H. Lindenblatt, F. Trost, K. Schnorr, S. Augustin, M. Braune, R. Treusch, T. Pfeifer, and R. Moshammer, Atomic, molecular and cluster science with the reaction microscope endstation at FLASH2, Appl. Sci. 10, 2953 (2020).

[22] G. Schmid, K. Schnorr, S. Augustin, S. Meister, H. Lindenblatt, F. Trost, Y. Liu, M. Braune, R. Treusch, C. D. Schröter, T. Pfeifer, and R. Moshammer, Reaction microscope endstation at FLASH2, J. Synchrotron Radiat. 26, 854 (2019).

[23] B. Faatz, E. Plönjes, S. Ackermann, A. Agababyan, V. Asgekar, V. Ayvazyan, S. Baark, N. Baboi, V. Balandin, N. von Bargen, Y. Bican, O. Bilani, J. Bödewadt, M. Böhnert, R. Böspflug, S. Bonfigt, H. Bolz, F. Borges, O. Borkenhagen, M. Brachmanski et al., Simultaneous operation of two soft x-ray free-electron lasers driven by one linear accelerator, New J. Phys. 18, 062002 (2016).

[24] W. Ackermann, G. Asova, V. Ayvazyan, A. Azima, N. Baboi, J. Bähr, V. Balandin, B. Beutner, A. Brandt, A. Bolzmann, R. Brinkmann, O. I. Brovko, M. Castellano, P. Castro et al., Operation of a free-electron laser from the extreme ultraviolet to the water window, Nat. Photon. 1, 336 (2007).

[25] B. Faatz, M. Braune, O. Hensler, K. Honkavaara, R. Kammering, M. Kuhlmann, E. Plönjes, J. RoenschSchulenburg, E. Schneidmiller, S. Schreiber et al., The FLASH facility: Advanced options for FLASH2 and future perspectives, Appl. Sci. 7, 1114 (2017).

[26] A. Kramida, Yu. Ralchenko, J. Reader, and NIST ASD Team, NIST Atomic Spectra Database (National Institute of Standards and Technology, Gaithersburg, MD, 2020, https://physics.nist. gov/asd.

[27] J. Ullrich, R. Moshammer, A. Dorn, R. Dörner, L. P. H. Schmidt, and H. Schmidt-Böcking, Recoil-ion and electron momentum spectroscopy: Reaction microscopes, Rep. Prog. Phys. 66, 1463 (2003).

[28] J. A. R. Samson and G. N. Haddad, Total photoabsorption cross sections of $\mathrm{H}_{2}$ from 18 to $113 \mathrm{eV}$, J. Opt. Soc. Am. B 11, 277 (1994).

[29] P. Birk, V. Stooß, M. Hartmann, G. D. Borisova, A. Blättermann, T. Heldt, K. Bartschat, C. Ott, and T. Pfeifer, Attosecond transient absorption of a continuum threshold, J. Phys. B: At., Mol. Opt. Phys. 53, 124002 (2020).

[30] N. Douguet, A. N. Grum-Grzhimailo, E. V. Gryzlova, E. I. Staroselskaya, J. Venzke, and K. Bartschat, Photoelectron angular distributions in bichromatic atomic ionization induced by circularly polarized VUV femtosecond pulses, Phys. Rev. A 93, 033402 (2016).

[31] M. B. Gaarde, C. Buth, J. L. Tate, and K. J. Schafer, Transient absorption and reshaping of ultrafast XUV light by laserdressed helium, Phys. Rev. A 83, 013419 (2011).

[32] W. L. Wiese and J. R. Fuhr, Accurate atomic transition probabilities for hydrogen, helium, and lithium, J. Phys. Chem. Ref. Data 38, 565 (2009).

[33] I. V. Hertel and C.-P. Schulz, Atome, Moleküle und optische Physik 1 (Springer, Berlin, 2017), p. 289.

[34] A. M. Bonch-Bruevich, N. N. Kostin, V. A. Khodovoi, and V. V. Khromov, Changes in the atomic absorption spectrum in the field of a light wave, Sov. Phys. JETP 29, 144 (1969).

[35] N. B. Delone and V. P. Krainov, AC Stark shift of atomic energy levels, Phys. Usp. 42, 669 (1999).

[36] U. Fano, Propensity rules: An analytical approach, Phys. Rev. A 32, 617 (1985).

[37] D. Busto, J. Vinbladh, S. Zhong, M. Isinger, S. Nandi, S. Maclot, P. Johnsson, M. Gisselbrecht, A. L'Huillier, E. Lindroth, and J. M. Dahlström, Fano's Propensity Rule in Angle-Resolved Attosecond Pump-Probe Photoionization, Phys. Rev. Lett. 123, 133201 (2019).

Correction: The fourth affiliation contained an error and has been fixed. 